The Astrophysical Journal, 617:575-579, 2004 December 10

(C) 2004. The American Astronomical Society. All rights reserved. Printed in U.S.A.

\title{
MULTIPLE COMPANIONS TO HD 154857 AND HD $160691^{1}$
}

\author{
Chris McCarthy, ${ }^{2,3}$ R. Paul Butler, ${ }^{2}$ C. G. Tinney, ${ }^{4}$ Hugh R. A. Jones, ${ }^{5}$ Geoffrey W. Marcy, ${ }^{3,6}$ \\ Brad Carter, ${ }^{7}$ Alan J. Penny, ${ }^{8}$ and Debra A. Fischer ${ }^{3,6}$ \\ Received 2004 June 10; accepted 2004 August 17
}

\begin{abstract}
Precise Doppler measurements from the Anglo-Australian Telescope (AAT) UCLES spectrometer reveal two companions to both HD 154857 and HD 160691. The inner companion to HD 154857 has a period of 398 days, an eccentricity of 0.51 , and a minimum mass of $1.8 M_{\mathrm{J}}$. An outer companion has a period much longer than $2 \mathrm{yr}$ and is currently detected only as a linear trend of $14 \mathrm{~m} \mathrm{~s}^{-1} \mathrm{yr}^{-1}$. The inner companion to HD 160691, previously announced from AAT data, has a period of 645 days, an eccentricity of 0.20 , and a minimum mass of $1.7 M_{\mathrm{J}}$. For the outer planet, whose orbit is less well constrained, a two-Keplerian fit yields a period of $8.2 \mathrm{yr}$, an eccentricity of 0.57 , and a minimum mass of $3.1 M_{\mathrm{J}}$. With these orbital parameters, its maximum separation from the star of 0.4 makes it a viable target for direct imaging.
\end{abstract}

Subject headings: planetary systems — stars: individual (HD 154857, HD 160691)

\section{INTRODUCTION}

All $\sim 110$ extrasolar planets ${ }^{9}$ discovered within 50 pc have been found from precision Doppler surveys over the past decade. It is these nearest stars with planets that are of the greatest interest for immediate follow-up by Hubble Space Telescope (HST) astrometry (Benedict et al. 2002) and spectroscopy (Charbonneau et al. 2002). Next-generation follow-up missions include precision astrometry with the Space Interferometry Mission and direct imaging of Jovian and terrestrial planets with giant ground-based telescopes such as the Thirty Meter Telescope (TMT; formerly CELT), ${ }^{10}$ Giant Segmented Mirror Telescope (GSMT), ${ }^{11}$ Giant Magellan Telescope,${ }^{12}$ and OverWhelmingly Large (OWL) telescope ${ }^{13}$ and space-based telescopes such as Terrestrial Planet Finder (TPF) and Darwin.

Precision Doppler surveys are most sensitive to planets orbiting Sun-like stars older than 2 Gyr that have no stellar companions within $3^{\prime \prime}$. Stars of spectral type earlier than late F rotate rapidly and have few absorption lines. Stars younger than 2 Gyr have photospheric magnetic fields and spots, which cause an intrinsic velocity "jitter" that correlates with chromospheric activity (Wright et al. 2004). Stars with close

\footnotetext{
${ }^{1}$ Based on observations obtained at the Anglo-Australian Telescope, Siding Spring, Australia.

2 Department of Terrestrial Magnetism, Carnegie Institution of Washington, 5241 Broad Branch Road NW, Washington, DC 20015-1305; paul@dtm.ciw. edu.

${ }^{3}$ Department of Physics and Astronomy, San Francisco State University, San Francisco, CA 94132.

4 Anglo-Australian Observatory, P.O. Box 296, Epping, NSW 1710, Australia.

5 Astrophysics Research Institute, Liverpool John Moores University, Twelve Quays House, Egerton Wharf, Birkenhead CH41 1LD, UK.

6 Department of Astronomy, University of California, Berkeley, CA 94720.

${ }^{7}$ Faculty of Sciences, University of Southern Queensland, Toowoomba, Queensland 4350, Australia.

${ }^{8}$ Rutherford Appleton Laboratory, Chilton, Didcot, Oxon, OX11 0QX, UK.

9 Count based on the list maintained at http://www.exoplanets.org as of 2004 June 10

10 The TMT Web site is http://tmt.ucolick.org.

11 For more information about GSMT, see http://www.aura-nio.noao.edu/ science.

${ }^{12}$ For more information about the Giant Magellan Telescope, see http:// www.gmto.org/.

${ }^{13}$ For more information about OWL, see http://www.eso.org/projects/owl/ index_3.html.
}

companions cannot be cleanly separated on a spectrometer entrance slit. Coincidentally, single stars that are older than 2 Gyr represent the ideal targets for habitable terrestrial planet searches, and by extension, searches for extraterrestrial intelligence (SETI; Turnbull \& Tarter 2003). There are roughly 2000 FGKM stars within $50 \mathrm{pc}$ that meet these criteria.

Over the past five years the number of stars being monitored by our precision Doppler programs has increased from $\sim 500$ to nearly all 2000 primary targets within $50 \mathrm{pc}$. We are carrying out northern hemisphere surveys with the Lick $3 \mathrm{~m}$ telescope (Fischer et al. 2003) and Keck I $10 \mathrm{~m}$ telescope (Vogt et al. 2002) and southern surveys with the Anglo-Australian Telescope (AAT) $3.9 \mathrm{~m}$ (Tinney et al. 2003) and the Magellan $6.5 \mathrm{~m}$ telescopes. These programs have yielded 75 of the 110 published extrasolar planets (Butler et al. 2002).

In 2002 the guaranteed allocation to our AAT planet search program was increased from 20 to 32 nights per year. In response, two changes were made. Sixty new stars were added, and observations with $\mathrm{S} / \mathrm{N}>200$ were demanded for all stars. In this paper we report two new planets from this survey, a planet around HD 154857, including evidence of a second, more distant companion, and a second planet around HD 160691.

\section{DOPPLER VELOCITIES AND KEPLERIAN FITS}

High-resolution spectra, $R \sim 50,000$, are taken with the UCLES echelle spectrometer (Diego et al. 1990) on the $3.9 \mathrm{~m}$ AAT. These spectra span the wavelength range from 48208550 Å. An iodine absorption cell (Marcy \& Butler 1992) provides wavelength calibration from 5000 to $6100 \AA$. The spectrometer point-spread function (PSF) is derived from the detailed shapes of the embedded iodine lines (Valenti et al. 1995; Butler et al. 1996).

Figure 1 shows four stable stars from the AAT. These observations span the time interval and the spectral types of the two planet-bearing stars reported in this paper. The Doppler analysis technique is outlined in Butler et al. (1996). Over the past year numerous improvements have been made to this algorithm. The long-term single-shot precision of chromospherically quiet $\mathrm{G}$ and $\mathrm{K}$ stars is currently $2 \mathrm{~m} \mathrm{~s}^{-1}$. Similar systems on the Lick $3 \mathrm{~m}$, Keck $10 \mathrm{~m}$, and VLT2 $8 \mathrm{~m}$ telescopes currently yield photon-limited precision of $3 \mathrm{~m} \mathrm{~s}^{-1}$ (Vogt et al. 2000; Butler et al. 2001, 2004; Marcy et al. 2005). 


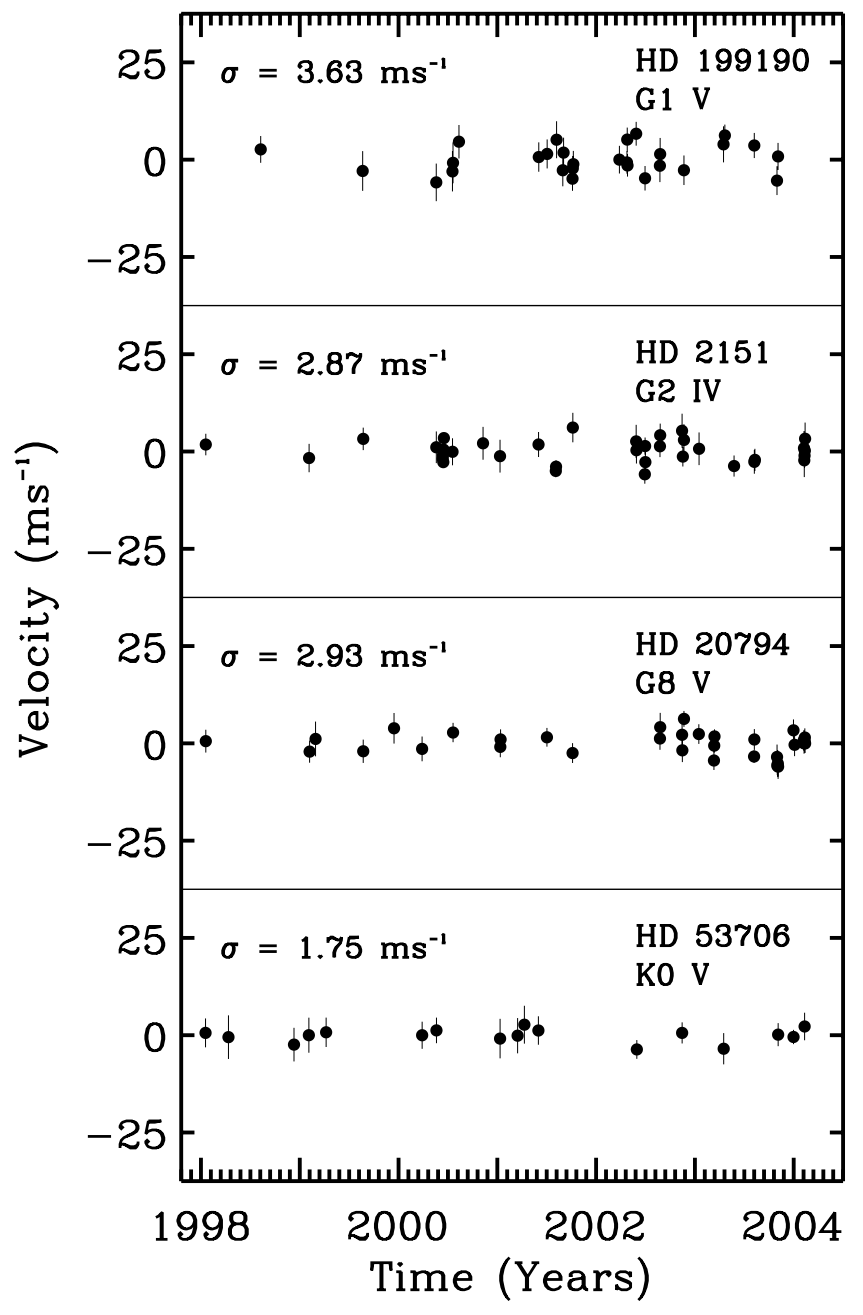

FIG. 1.-AAT Doppler velocities of chromospherically quiet $\mathrm{G}$ and $\mathrm{K}$ dwarfs and subgiants. These observations span the full six years of the AAT Planet Search Project.

\section{1. $H D 154857$}

Houk \& Cowley (1975) classify HD 154857 (HIP 84069, SAO 244491) as a G5 dwarf, consistent with its color, $B-V=$ 0.65. The Hipparcos-derived distance, 68.5 pc (ESA 1997; Perryman et al. 1997), yields an absolute $V$ magnitude, $M_{V}=$ $3.07, \sim 2$ mag above the main sequence, suggesting that the star is evolved. High metallicity alone is unlikely to produce such a large displacement from the main sequence. Like most evolved stars moving toward subgiant status, HD 154857 is chromospherically quiet with $\log R^{\prime} H K=-5.14$ (Henry et al. 1996) but not in a Maunder minimum state (Wright 2004). The star is photometrically stable with a scatter of 0.008 mag, consistent with Hipparcos measurement error (ESA 1997).

Spectral synthesis matched to the spectrum of HD 154857 (Fischer \& Valenti 2004) yields $T_{\text {eff }}=5628 \pm 40 \mathrm{~K}, \log g=$ $4.06 \pm 0.05$, and $V \sin i=3.31 \pm 0.3 \mathrm{~km} \mathrm{~s}^{-1}$, consistent with a G5 dwarf evolving toward subgiant status. The derived metallicity, $[\mathrm{Fe} / \mathrm{H}]=-0.23 \pm 0.03$, is slightly lower than that for typical field stars (Reid 2002). Interpolation on the grid of stellar models of Girardi et al. (2002) yields a stellar mass of $1.17 \pm 0.05 M_{\odot}$ and an age of 5 Gyr.

A total of 18 observations of HD 154857, taken between 2002 April and 2004 February, are shown in Figure 2 and listed in Table 1. The median value of internal measurement uncertainties is $3.39 \mathrm{~m} \mathrm{~s}^{-1}$. The dashed line shows the best-fit

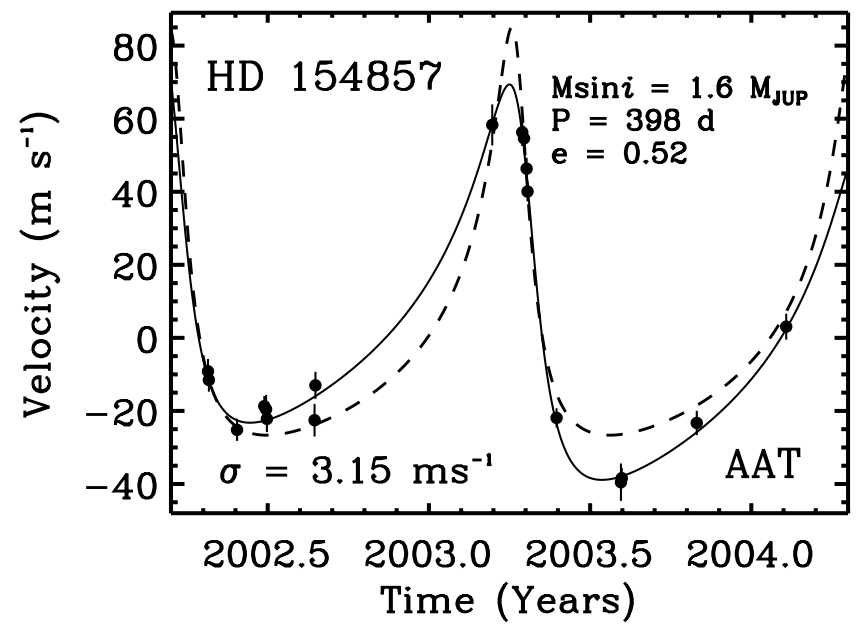

FIG. 2.-Doppler velocities for HD 154857. The dashed line is the best-fit Keplerian. The rms of $7.66 \mathrm{~m} \mathrm{~s}^{-1}$ is significantly worse than the median measurement uncertainty of $3.39 \mathrm{~m} \mathrm{~s}^{-1}$. The solid line shows the best-fit Keplerian model with an additional linear trend. The rms of $3.15 \mathrm{~m} \mathrm{~s}^{-1}$ is consistent with measurement uncertainty. The period of this Keplerian model is 398.5 days, the semiamplitude is $52 \mathrm{~m} \mathrm{~s}^{-1}$, and the eccentricity is 0.51 , yielding a minimum mass of $1.80 \mathrm{M}_{\mathrm{J}}$ and a semimajor axis of $1.11 \mathrm{AU}$. The linear trend is presumably due to a companion with a period much longer than $2 \mathrm{yr}$ and a mass significantly greater than $M_{\mathrm{J}}$.

single-Keplerian model. The rms to this fit is $7.66 \mathrm{~m} \mathrm{~s}^{-1}$, yielding $\chi_{\nu}=2.21$. The poor quality of this fit motivated the addition of a linear trend to the Keplerian. The solid line shows the resulting best-fit Keplerian plus linear trend. The rms to this fit is $3.15 \mathrm{~m} \mathrm{~s}^{-1}$, yielding $\chi_{\nu}=0.87$. The period of the Keplerian is 398.5 days, the semiamplitude is $52 \mathrm{~m} \mathrm{~s}^{-1}$, and the eccentricity is 0.51 , yielding a minimum $(M \sin i)$ mass of $1.80 M_{\odot}$ and a semimajor axis of $1.11 \mathrm{AU}$. The linear trend of $-14 \mathrm{~m} \mathrm{~s}^{-1} \mathrm{yr}^{-1}$ is presumably due to an additional companion with a period much longer than $2 \mathrm{yr}$ and a semiamplitude greater than $14 \mathrm{~m} \mathrm{~s}^{-1}$. Astrometry from Hipparcos reveals no wobble at the level of 1 mas (ESA 1997). This places no useful constraint on the outer companion as its orbital period is longer than the Hipparcos mission duration of $3 \mathrm{yr}$, allowing any

TABLE 1

Velocities FOR HD 154857

\begin{tabular}{|c|c|c|}
\hline $\begin{array}{c}\text { JD } \\
(-2,450,000)\end{array}$ & $\begin{array}{l}\text { Radial Velocity } \\
\qquad\left(\mathrm{m} \mathrm{s}^{-1}\right)\end{array}$ & $\begin{array}{c}\text { Error } \\
\left(\mathrm{m} \mathrm{s}^{-1}\right)\end{array}$ \\
\hline $2389.2358 \ldots \ldots \ldots \ldots \ldots \ldots . .$. & -14.6 & 3.4 \\
\hline $2390.2122 \ldots \ldots \ldots \ldots \ldots \ldots$ & -16.9 & 3.2 \\
\hline $2422.1371 \ldots \ldots \ldots \ldots \ldots . .$. & -30.6 & 3.0 \\
\hline $2453.0198 \ldots \ldots \ldots \ldots \ldots$ & -24.1 & 2.8 \\
\hline $2455.0253 \ldots \ldots \ldots \ldots \ldots \ldots$ & -25.0 & 3.9 \\
\hline $2455.9766 \ldots \ldots \ldots \ldots \ldots \ldots$ & -27.6 & 3.6 \\
\hline $2509.9485 \ldots \ldots \ldots \ldots \ldots \ldots$ & -28.0 & 4.5 \\
\hline $2510.9162 \ldots \ldots \ldots \ldots \ldots$ & -18.4 & 3.7 \\
\hline $2711.2461 \ldots \ldots \ldots \ldots \ldots$ & 52.9 & 5.6 \\
\hline $2745.2427 \ldots \ldots \ldots \ldots \ldots \ldots$ & 50.8 & 3.7 \\
\hline $2747.2115 \ldots \ldots \ldots \ldots \ldots \ldots$ & 49.2 & 3.4 \\
\hline $2750.1777 \ldots \ldots \ldots \ldots \ldots \ldots$ & 40.9 & 3.0 \\
\hline $2751.2291 \ldots \ldots \ldots \ldots \ldots \ldots$ & 34.6 & 2.7 \\
\hline $2784.1264 \ldots \ldots \ldots \ldots \ldots$ & -27.3 & 2.6 \\
\hline $2857.0297 \ldots \ldots \ldots \ldots \ldots$ & -44.9 & 5.1 \\
\hline 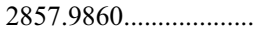 & -43.8 & 2.8 \\
\hline $2942.9120 \ldots \ldots \ldots \ldots \ldots$ & -28.7 & 3.3 \\
\hline 3044.2691..................... & -2.4 & 3.5 \\
\hline
\end{tabular}


TABLE 2

VELOCITIES FOR HD 160691

\begin{tabular}{|c|c|c|}
\hline $\begin{array}{c}\text { JD } \\
(-2,450,000)\end{array}$ & $\begin{array}{l}\text { Radial Velocity } \\
\qquad\left(\mathrm{m} \mathrm{s}^{-1}\right)\end{array}$ & $\begin{array}{c}\text { Error } \\
\left(\mathrm{m} \mathrm{s}^{-1}\right)\end{array}$ \\
\hline $915.2911 \ldots \ldots$. & -18.8 & 5.1 \\
\hline 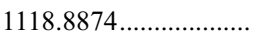 & -4.9 & 2.7 \\
\hline $1119.9022 \ldots \ldots \ldots \ldots \ldots . .$. & -9.9 & 2.4 \\
\hline 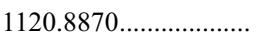 & -8.7 & 2.4 \\
\hline $1121.8928 \ldots \ldots \ldots \ldots \ldots$ & -8.0 & 2.6 \\
\hline 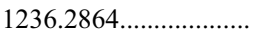 & -35.4 & 3.6 \\
\hline $1410.8977 \ldots \ldots \ldots \ldots \ldots \ldots$ & -47.8 & 2.4 \\
\hline $1413.8981 \ldots \ldots \ldots \ldots \ldots \ldots$ & -43.0 & 2.2 \\
\hline $1630.3042 \ldots \ldots \ldots \ldots \ldots \ldots$ & 36.0 & 2.8 \\
\hline 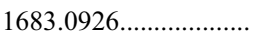 & 41.4 & 2.9 \\
\hline 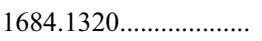 & 38.4 & 3.5 \\
\hline 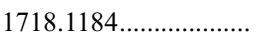 & 26.5 & 3.1 \\
\hline $1742.9096 \ldots \ldots \ldots \ldots \ldots$ & 13.4 & 2.8 \\
\hline 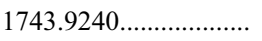 & 24.0 & 3.1 \\
\hline $1745.0440 \ldots \ldots \ldots \ldots \ldots \ldots$ & 14.5 & 2.8 \\
\hline 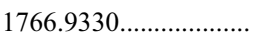 & 9.9 & 2.3 \\
\hline $1767.9689 \ldots \ldots \ldots \ldots \ldots \ldots$ & 8.7 & 2.5 \\
\hline $1827.8973 \ldots \ldots \ldots \ldots$ & -5.0 & 2.4 \\
\hline $1828.8866 \ldots \ldots \ldots \ldots \ldots \ldots$ & -2.3 & 3.1 \\
\hline $1829.8890 \ldots \ldots \ldots \ldots \ldots \ldots$ & -2.4 & 3.4 \\
\hline $1855.9058 \ldots \ldots \ldots \ldots \ldots \ldots$ & -10.7 & 4.4 \\
\hline 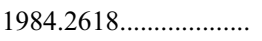 & -25.1 & 3.1 \\
\hline 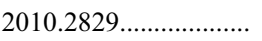 & -30.8 & 5.3 \\
\hline 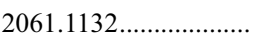 & -29.2 & 2.9 \\
\hline $2091.9807 \ldots \ldots \ldots \ldots \ldots . .$. & -28.0 & 2.6 \\
\hline $2126.9766 \ldots \ldots \ldots \ldots \ldots$ & -13.1 & 2.9 \\
\hline 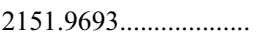 & -2.7 & 2.7 \\
\hline 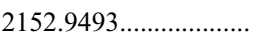 & -2.9 & 2.3 \\
\hline 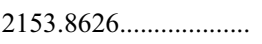 & 2.8 & 2.6 \\
\hline $2186.9095 \ldots \ldots \ldots \ldots \ldots . .$. & 18.1 & 2.2 \\
\hline $2187.8879 \ldots \ldots \ldots \ldots \ldots$ & 19.5 & 2.4 \\
\hline $2360.3245 \ldots \ldots \ldots \ldots \ldots$ & 28.7 & 2.2 \\
\hline 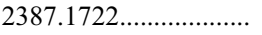 & 29.2 & 2.3 \\
\hline 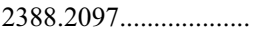 & 25.9 & 2.4 \\
\hline 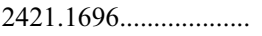 & 10.9 & 2.2 \\
\hline $2425.1226 \ldots \ldots \ldots \ldots \ldots$ & 18.3 & 2.8 \\
\hline $2455.0437 \ldots \ldots \ldots \ldots \ldots . .$. & 10.6 & 2.8 \\
\hline $2509.9587 \ldots \ldots \ldots \ldots \ldots$ & 4.0 & 3.0 \\
\hline 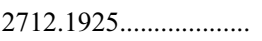 & -42.4 & 3.5 \\
\hline 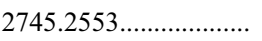 & -22.7 & 2.5 \\
\hline $2752.1799 \ldots \ldots \ldots \ldots \ldots \ldots$ & -20.7 & 2.8 \\
\hline $2785.1422 \ldots \ldots \ldots \ldots \ldots \ldots$ & -1.8 & 2.8 \\
\hline 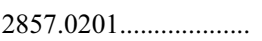 & 27.3 & 1.8 \\
\hline 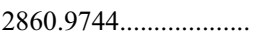 & 25.2 & 2.6 \\
\hline 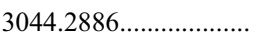 & 14.0 & 2.9 \\
\hline
\end{tabular}

astrometric wobble to be absorbed into the solution for proper motion.

\section{2. $H D 160691$}

The Bright Star Catalogue (Hoffleit \& Jaschek 1982) assigns a spectral type of G3 IV-V to HD 160691 (HR 6585, HIP 86796, GL 691, Mu Ara), in reasonable agreement with numerous other determinations (see SIMBAD) that typically yield G5 V. The Hipparcos distance of HD 160691 is 15.3 pc, yielding an absolute magnitude of $M_{V}=4.23$. The star is photometrically stable within Hipparcos measurement error, exhibiting photometric scatter of $0.002 \mathrm{mag}$, and is chromospherically inactive, with $\log R^{\prime}(H K)=-5.02$ (Henry et al. 1996).

Spectral synthesis models fitted to our high-resolution spectrum of HD 160691 (Fischer \& Valenti 2004) yield $T_{\text {eff }}=$ $5807 \pm 30 \mathrm{~K}, \log g=4.37 \pm 0.05$, and $V \sin i=4.07 \pm 0.3 \mathrm{~km}$

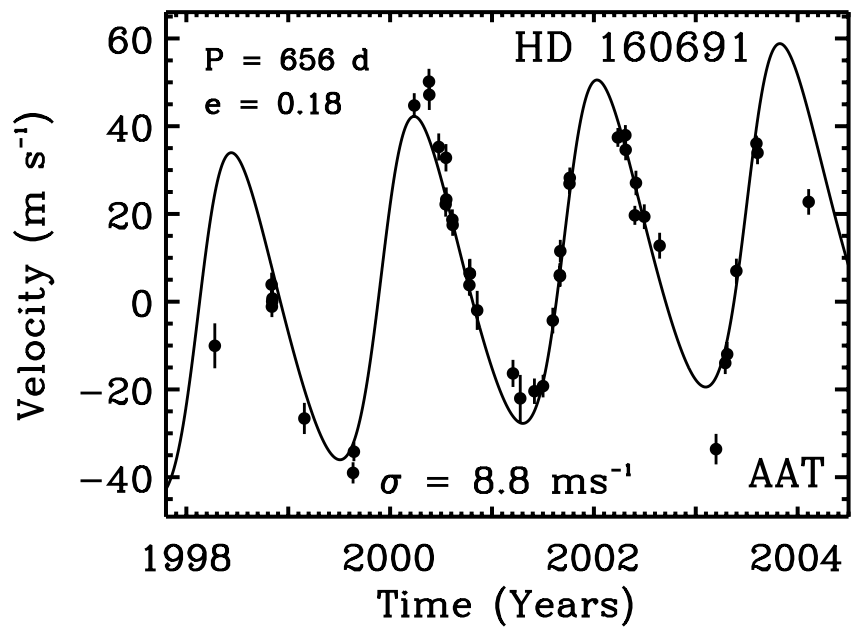

FIG. 3.-Doppler velocities for HD 160691. The solid line is the best-fit Keplerian plus linear trend. The rms to this model is $8.84 \mathrm{~m} \mathrm{~s}^{-1}$, significantly worse than the median internal measurement uncertainty of $2.76 \mathrm{~m} \mathrm{~s}^{-1}$.

$\mathrm{s}^{-1}$. The derived metallicity, $[\mathrm{Fe} / \mathrm{H}]=+0.263 \pm 0.03$, is consistent with previous determinations (Favata et al. 1997; Bensby et al. 2003). Interpolation on the grid of stellar models by Girardi et al. (2002) yields a stellar mass of $1.08 \pm 0.05 M_{\odot}$ and an age of 6 Gyr.

Based on 2 yr of AAT data, Butler et al. (2001) reported a planet with a $2 \mathrm{yr}$ period orbiting HD 160691. With $4 \mathrm{yr}$ of data, Jones et al. (2002) confirmed this planet and found an additional linear trend. Table 2 lists the 45 observations of HD 160691 that now span 5.8 yr. These measurements, shown in Figure 3 , have a median internal uncertainty of $2.76 \mathrm{~m} \mathrm{~s}^{-1}$. The rms to the best-fit Keplerian model plus a linear trend (solid line) is $8.84 \mathrm{~m} \mathrm{~s}^{-1}$. With $\chi_{\nu}=2.60$, this model no longer adequately fits the data.

Figure 4 shows a double-Keplerian fit to the data. The periods of these two Keplerian models are 1.76 and $8.17 \mathrm{yr}$, with orbital eccentricities of 0.20 and 0.57 , semiamplitudes of 38 and $51 \mathrm{~m} \mathrm{~s}^{-1}$, minimum $(M \sin i)$ masses of $1.67 M_{\mathrm{J}}$ and $3.10 M_{\mathrm{J}}$,

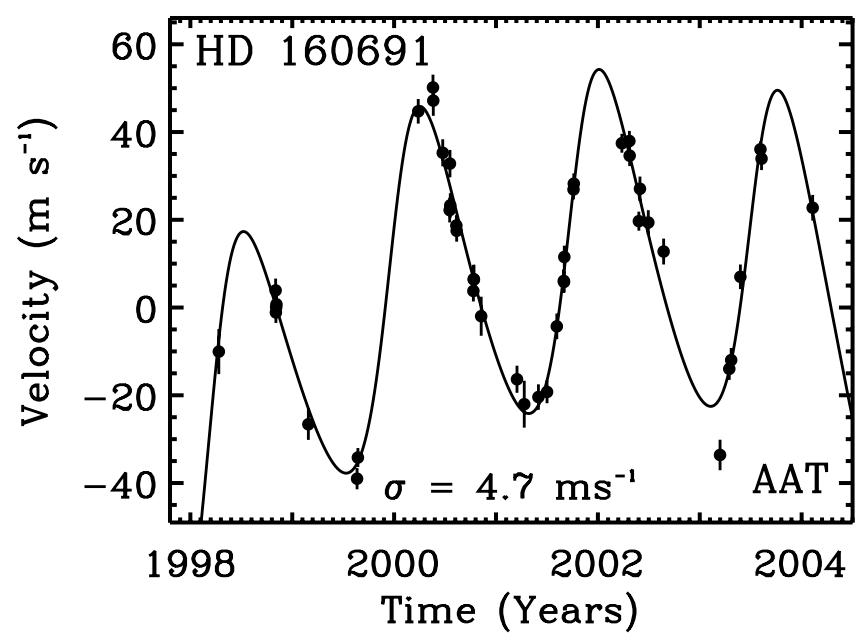

FIG. 4.-A double Keplerian fit to HD 160691. The inner Keplerian has a period of 645 days, an eccentricity of 0.20 , and a semiamplitude of $38 \mathrm{~m} \mathrm{~s}^{-1}$, yielding a minimum mass of $1.68 M_{\mathrm{J}}$ and a semimajor axis of $1.50 \mathrm{AU}$. The outer Keplerian has a period of $8.2 \mathrm{yr}$, an eccentricity of 0.57 , and a semiamplitude of $51 \mathrm{~m} \mathrm{~s}^{-1}$, yielding a minimum mass of $3.1 M_{\mathrm{J}}$ and a semimajor axis of $4.16 \mathrm{AU}$. The rms to this model is $4.66 \mathrm{~m} \mathrm{~s}^{-1}(1.69 \sigma)$. 
TABLE 3

Orbital Parameters

\begin{tabular}{|c|c|c|c|}
\hline Parameter & HD $154857^{\mathrm{a}}$ & HD 160691b & HD $160691 \mathrm{c}$ \\
\hline Orbital period $P$ (days) .......... & $398.5 \pm 9$ & $645.5 \pm 3$ & $2986 \pm 30$ \\
\hline Velocity amplitude $K\left(\mathrm{~m} \mathrm{~s}^{-1}\right) \ldots \ldots \ldots$ & $52 \pm 5$ & $38 \pm 2$ & $51 \pm 7$ \\
\hline 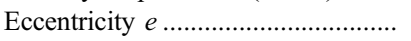 & $0.51 \pm 0.06$ & $0.20 \pm 0.03$ & $0.57 \pm 0.1$ \\
\hline$\omega(\operatorname{deg})$ & $50 \pm 11$ & $294 \pm 9$ & $161 \pm 8$ \\
\hline 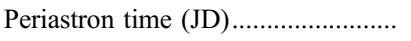 & $2,451,963 \pm 10$ & $2,450,645.5 \pm 4$ & $2,450,541 \pm 96$ \\
\hline$M \sin i\left(M_{\mathrm{J}}\right) \ldots \ldots \ldots \ldots \ldots \ldots \ldots \ldots \ldots \ldots \ldots \ldots \ldots \ldots \ldots \ldots \ldots \ldots \ldots$ & $1.80 \pm 0.27$ & $1.67 \pm 0.11$ & $3.10 \pm 0.71$ \\
\hline Semimajor axis $a(\mathrm{AU})$ & $1.11 \pm 0.02$ & $1.50 \pm 0.02$ & $4.17 \pm 0.07$ \\
\hline $\mathrm{rms}\left(\mathrm{m} \mathrm{s}^{-1}\right)$ & 3.15 & 4.66 & $\ldots$ \\
\hline
\end{tabular}

NoтE.-Errors in fit parameters $(1 \sigma)$ are derived from formal Monte Carlo tests. Error in the derived quantities $M \sin i$ and $a$ reflect the quadrature sum of $1 \sigma$ errors in fit Keplerian parameters and stellar mass.

${ }^{a}$ Additional slope is $-14.3 \pm 3.5 \mathrm{~m} \mathrm{~s}^{-1} \mathrm{yr}^{-1}$.

and semimajor axes of 1.50 and $4.16 \mathrm{AU}$, respectively. This double-Keplerian model represents a significant improvement over the single-Keplerian model with a linear trend, with residuals yielding $\mathrm{rms}=4.66 \mathrm{~m} \mathrm{~s}^{-1}$ and $\chi_{\nu}=1.60$. Nonetheless, other fits with somewhat different periods and eccentricities may have only slightly worse values of rms scatter and $\chi_{\nu}$. Multi-Keplerian best fits can occasionally yield solutions that are not dynamically stable (Gozdziewski et al. 2003). Sophisticated dynamical analyses of this system, such as those that impose the assumption of orbital stability, which employ Jacobi coordinates (e.g., Lee \& Peale 2003; Gozdziewski et al. 2003), and those that use genetic algorithms to derive superior initial guesses are warranted but beyond the scope of the present paper. Table 3 shows the orbital parameters for these two planets as well as those of the companion to HD 154857.

Figure 5 shows each of the Keplerian orbits separately. While the period of the inner planet is tightly constrained with more than three full orbits observed, the outer planet is somewhat underconstrained with observations spanning only $70 \%$ of the full period. Hipparcos astrometry reveals no wobble above standard errors of $\sim 1$ mas. The outer companion would cause a wobble easily absorbed into the solution for proper motion.

Based on these fits, the maximum angular separation of the outer planet, HD $160691 \mathrm{c}$, from the star is expected to be $\alpha=$

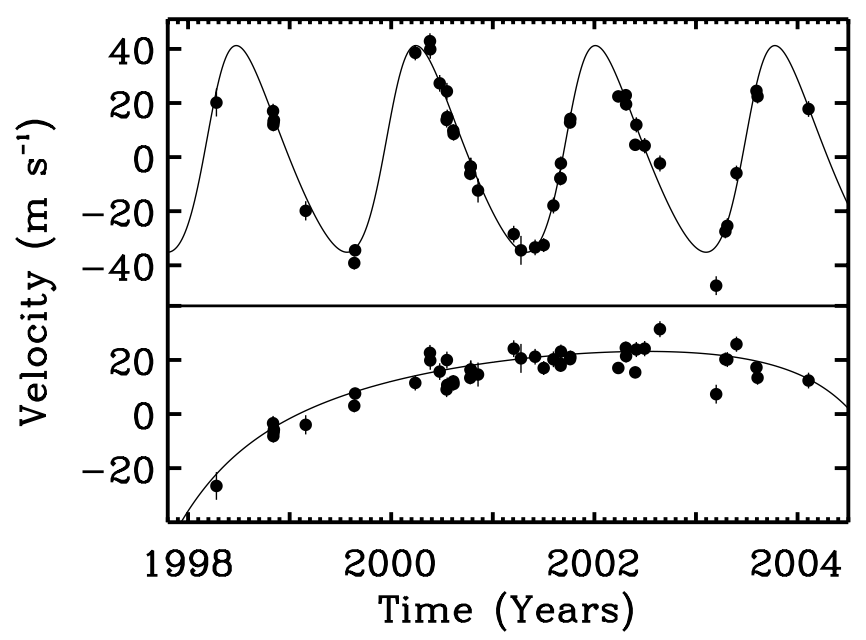

FIG. 5.-Individual Keplerian models to HD 160691. Each of the two Keplerian models from Fig. 4 is shown separately. The top figure shows the 645 day Keplerian, while the bottom figure shows the 8.17 yr Keplerian. Approximately $70 \%$ of the outer Keplerian has been observed. $a(1+e) / d=0$ ".43. This planet is detectable by future imaging systems that achieve resolution $(\sim 4 \lambda / d)$ of $<0$ " 4 and that carefully suppresses the wings of the PSF due to mirror roughness, including proposed space-based programs such as Eclipse (Ealey \& Trauger 2004) and ground-based efforts at $\mathrm{VLT}^{14}$ and Gemini. $^{15}$

Future astrometry with precision of better than 1 mas, such as that offered by HST and its Fine Guidance Sensor (Benedict et al. 2002) or by the Space Interferometry Mission (SIM), can also detect HD 160691c; however, a duration of $\sim 8$ yr would be required.

\section{DISCUSSION}

Multiple-planet systems are revealed considerably more slowly than single planets. For "hierarchical" systems with widely separated planets, the inner planet usually induces the largest Doppler wobble in the host star, making it typically detectable first, as occurred with 55 Cnc (Marcy et al. 2002) and $v$ And (Butler et al. 1999, 1997). For cases where the periods of both planets are short, the most massive planet may be revealed first, as with GL 876 (Marcy et al. 2001). Detectability stems from both the amplitude of the Doppler signal and the number of cycles that have transpired during the string of observations.

Both of the systems reported in this paper are of the first type, with the inner planet detected first. For the case of HD 154857 , the presumed outer companion is detected only as a linear trend at this time. Similarly, the existence of the second companions to HD 160691 and to $55 \mathrm{Cnc}$ were first reported as a linear trends (e.g., Jones et al. 2002).

Based on the results of Jones et al. (2002), Bois et al. (2003) carried out a study of the dynamic stability of the HD 160691 system. They concluded that the two planets must be in a $2: 1$ mean-motion resonance and that the outer companion should be in a highly eccentric $(e>0.52)$ orbit. With the identical data set, Gozdziewski et al. (2003) concluded that (1) this system cannot be in a 2:1 mean-motion resonance, (2) if the semimajor axis of the outer companion is smaller than 5.2 AU the system should be in a low-order mean-motion resonance (" $3: 1,7: 2,4: 1,5: 1$, or to their vicinity"), and (3) the eccentricity of the outer companion should be $<0.5$.

With data covering only $\sim 70 \%$ of the orbit, the orbital parameters of the outer companion to HD 160691 have relatively

\footnotetext{
14 See http://www.eso.org/instruments.

15 See http://www.gemini.edu/science.
} 
large uncertainties. Nonetheless, the lower limit of the period of HD 160691c is $8 \mathrm{yr}$, ruling out a $2: 1$ mean-motion resonance with the inner planet $(P=1.76 \mathrm{yr})$. Similarly, meanmotion resonances of $3: 1$ and $4: 1$ seem to be ruled out. A $5: 1$ mean-motion resonance remains plausible as is one of $9: 2$. The orbital eccentricity of the outer planet, however, remains poorly constrained.

Both of these systems highlight the importance of long-term monitoring and of Doppler measurement precision. Neither the second planet to HD 160691 nor the linear trend to HD 154857 would be detectable with measurement uncertainties $>7 \mathrm{~m} \mathrm{~s}^{-1}$. The outer planet around HD 160691 is one of only a few known extrasolar planets that is separated from its host star at apastron by over 0 ". 4 , making it a prime target for future efforts designed to directly image extrasolar planets.
We gratefully acknowledge the superb technical support at the Anglo-Australian Telescope, which has been critical to the success of this project, in particular E. Penny, R. Paterson, D. Stafford, F. Freeman, S. Lee, J. Pogson, and G. Schaffer. We further acknowledge the UK and Australian government support of the Anglo-Australian Telescope through their PPARC and DETYA funding to H. R. A. J., A. J. P., and C. G. T. We received support from NSF grant AST-9988087 and travel support from the Carnegie Institution of Washington (to R. P. B.), NASA grant NAG5-8299, and NSF grant AST95-20443 (to G. W. M.), and from Sun Microsystems. We thank the Australian and UK Telescope assignment committees (ATAC \& PATT) for allocations of telescope time. This research has made use of NASA's Astrophysics Data System and the SIMBAD database, operated at CDS, Strasbourg, France.
Benedict, G. F., et al. 2002, ApJ, 581, L115

Bensby, T., Feltzing, S., \& Lundstroem, I. 2003, A\&A, 410, 527

Bois, E., Kiseleva-Eggleton, L., Rambaux, N., \& Pilat-Lohinger, E. 2003, ApJ, 598,1312

Butler, R. P., Bedding, T. R., Kjeldsen, H., McCarthy, C., O’Toole, S. J., Tinney, C. G., Marcy, G. W., \& Wright, J. T. 2004, ApJ, 600, L75

Butler, R. P., Marcy, G. W., Fischer, D. A., Brown, T. M., Contos, A. R., Korzennik, S. G., Nisenson, P., \& Noyes, R. W. 1999, ApJ, 526, 916

Butler, R. P., Marcy, G. W., Williams, E., Hauser, H., \& Shirts, P. 1997, ApJ, 474, L115

Butler, R. P., Marcy, G. W., Williams, E., McCarthy, C., Dosanjh, P., \& Vogt, S. S. 1996, PASP, 108, 500

Butler, R. P., Tinney, C. G., Marcy, G. W., Jones, H. R. A., Penny, A. J., \& Apps, K. 2001, ApJ, 555, 410

Butler, R. P., et al. 2002, ApJ, 578, 565

Charbonneau, D., Brown, T. M., Noyes, R. W., \& Gilliland, R. L. 2002, ApJ, 568,377

Diego, F., Charalambous, A., Fish, A. C., \& Walker, D. D. 1990, Proc. SPIE, 1235,562

Ealey, M. A., \& Trauger, J. T. 2004, in Proc. SPIE, 5166, 172

ESA. 1997, Hipparcos and Tycho Catalogues (Garching: ESA), 1239

Favata, F., Micela, G., \& Sciortino, S. 1997, A\&A, 323, 809

Fischer, D. A., \& Valenti, J. 2004, ApJ, submitted

Fischer, D. A., et al. 2003, ApJ, 586, 1394

Girardi, L., Bertelli, G., Bressan, A., Chiosi, C., Groenewegen, M. A. T., Marigo, P., Salasnich, B., \& Weiss, A. 2002, A\&A, 391, 195

Gozdziewski, K., Konacki, M., \& Maciejewski, A. J. 2003, ApJ, 594, 1019

\section{REFERENCES}

Henry, T. J., Soderblom, D. R., Donahue, R. A., \& Baliunas, S. L. 1996, AJ, 111,439

Hoffleit, D., \& Jaschek, C. 1982, The Bright Star Catalogue (4th ed.; New Haven: Yale Univ.)

Houk, N., \& Cowley, A. P. 1975, Michigan Catalogue of Two-Dimensional Spectral Types for the HD Stars (Ann Arbor: Univ. Michigan)

Jones, H. R. A., Butler, R. P., Marcy, G. W., Tinney, C. G., Penny, A. J., McCarthy, C., \& Carter, B. D. 2002, MNRAS, 337, 1170

Lee, M. H., \& Peale, S. J. 2003, ApJ, 592, 1201

Marcy, G. W., \& Butler, R. P. 1992, PASP, 104, 270

Marcy, G. W., Butler, R. P., Fischer, D. A., Laughlin, G., Vogt, S. S., Henry, G. W., \& Pourbaix, D. 2002, ApJ, 581, 1375

Marcy, G. W., Butler, R. P., Fischer, D. A., Vogt, S. S., Lissauer, J. J., \& Rivera, E. J. 2001, ApJ, 556, 296

Marcy, G. W., Butler, R. P., Vogt, S. S., Fischer, D. A., Henry, G. W., Laughlin, G., Wright, J. T., \& Johnson, J. 2005, ApJ, in press

Perryman, M. A. C., et al. 1997, A\&A, 323, L49

Reid, I. N. 2002, PASP, 114, 306

Tinney, C. G., Butler, R. P., Marcy, G. W., Jones, H. R. A., Penny, A. J., McCarthy, C., Carter, B. D., \& Bond, J. 2003, ApJ, 587, 423

Turnbull, M. C., \& Tarter, J. C. 2003, ApJS, 145, 181

Valenti, J., Butler, R. P., \& Marcy, G. W. 1995, PASP, 107, 966

Vogt, S. S., Butler, R. P., Marcy, G. W., Fischer, D. A., Dimitri, P., Apps, K., \& Laughlin, G. 2002, ApJ, 568, 352

Vogt, S. S., Marcy, G. W., Butler, R. P., \& Apps, K. 2000, ApJ, 536, 902

Wright, J. T. 2004, AJ, 128, 1273

Wright, J. T., Marcy, G. W., Butler, R. P., \& Vogt, S. S. 2004, ApJS, 152, 261 